\title{
Understanding Macroscopic Human Behavior
}

\author{
Ramesh Jain \\ Department of Computer Science \\ University of California, Irvine \\ Irvine, CA 92697 \\ jain@ics.uci.edu
}

\begin{abstract}
The Web has changed the way we live, work, and socialize. Web-thinking has been influencing how we understand, design, and solve important societal problems and build complex systems. For centuries, emergence has been considered an essential property underlying the way complex systems and patterns emerge out of relatively simple interactions among different components. The Web has compellingly demonstrated results of emergence in understanding human behavior not at an individual level but at different macro levels ranging from social networks to global levels. Recent rapid advances in sensor technology, Web 2.0, Mobile devices, and Web technologies have opened further opportunities to understand macroscopic human behavior. In this talk, we will discuss our approach to build a framework for studying macroscopic human behavior based on micro-events including Tweets and other participatory sensing approaches.
\end{abstract}

